\title{
La pourriture brune des cabosses du cacaoyer au Cameroun: mise en évidence d'un caractère de résistance au champ
}

\author{
D. Despréaux ${ }^{1}$, D. Clément ${ }^{2}$ et M. Partiot ${ }^{3}$ \\ 1 INRACIRAD, station de pathologie végétale, route de Saint Cyr, 78026 Versailles Cedex, France; \\ ${ }^{2}$ CIRAD, station IRCC de Bingerville, 01 BP 1827, Abidjan, Côte d'Ivoire; \\ 3 CIRAD, IRA, BP 2123, Yaoundé, Cameroun
}

(reçu le 03 février 1989, accepté le 13 avril 1989)

Résumé - Cette étude porte sur l'analyse de l'évolution naturelle de la pourriture brune des cabosses pendant 3 années consécutives, dans un essai diallèle $6 \times 6$ quasi complet (sans les autofécondations) installé en 1974 dans la station de Barombi-kang.

Les résultats obtenus révèlent l'existence d'un caractère de résistance partielle au champ génétiquement transmissible. Les pourcentages de pertes sont significativement moins importants parmi les descendances du clone UPA134 que parmi les autres familles. A un moindre niveau, un phénomène similaire est observé pour celles du clone SNK413.

Par ailleurs, les croisements avec le clone UPA134 donnent les meilleures productions. Les transmissions des caractères liés à la résistance et à la productivité sont indépendantes et se font principalement de façon additive.

cabosses - cacaoyers - Cameroun - pourriture brune - résistance

Summary - Black pod disease of cocoa in Cameroun: analysis of a resistant character observed in field. This study analyses the natural evolution of the black pod disease for 3 years, in a diallel $6 \times 6$ trial of cocoa, established in Barombi-kang experimental station in 1974. The variance analyses are carried out on the potential yield (ripe and black pods), on the effective yield (ripe pods) and on the losses (percentage of black pods).

The results show the existence of a genetic form of resistance in field conditions. The loses are less important for the clone UPA134 progenies than for the other families. The same phenomenom is also observed to a lesser extent for the clone SNK413. The UPA134 progenies are also the best pod producers. Nevertheless, the transmission of resistance and productivity does not seem to be linked. Both characters are of an additive type. The origin of the resistance character is discussed.

pods - cocoa - Cameroun - black pod disease - resistance

\section{INTRODUCTION}

La pourriture brune des cabosses peut être provoquée par plusieurs Phytophthora sp. (Griffin, 1977), mais au Cameroun l'espèce parasite dominante est Phytophthora megakarya (Bakala, 1979). Ce parasite se développe essentiellement dans les fruits pendant les périodes pluvieuses, entre mai et décembre. Les modalités de propagation de la maladie ne sont pas encore connues avec certitude, mais il est probable que pendant les périodes favorables à l'épidémie les fortes pluies soient à l'origine de la formation de brouillards infectieux très volatiles (Gregory \& Maddisson, 1984). Ainsi, dès le début de l'épidémie, tous les fruits présents dans une plantation sont soumis à la pression de la maladie. Celle-ci est cependant plus forte sur les fruits situés dans les parties basses des arbres.

Au Cameroun, la pourriture brune peut entraîner la perte quasi totale de la récolte. Les Services de vulgarisation préconisent des applications périodiques de fongicide, mais ces mesures de protection sont encore insuffisamment efficaces (Despréaux et al., 1987). 
L'étude de la résistance génétique de Théobroma cacao L. aux Phytophthora spp. a déjà fait l'objet de nombreux travaux. Aucun cas de résistance totale n'ayant été relevé, les recherches se sont surtout orientées vers une évaluation de la résistance partielle des différents clones connus, à la suite d'infections artificielles pratiquées sur d'autres organes que les fruits (Amponsah \& Asare Nyako, 1973; Partiot, 1975; Tarjot, 1977). Cependant, les résultats les plus intéressants ont été obtenus à la suite d'infections artificielles pratiquées directement sur les cabosses. Ainsi, Blaha \& Lotode (1976) ont montré l'existence d'une forme de résistance des fruits à la pénétration et à la progression du parasite dans les tissus. Ces observations, qui ont été partiellement confirmées par la suite (Lawrence, 1978; Nwaga, 1984), ont été à l'origine de l'installation d'un essai diallèle en 1974 au Cameroun.

L'étude présentée porte sur l'analyse du comportement de cet essai diallèle face à la pourriture brune des cabosses en conditions naturelles d'infection.

\section{MATÉRIEL ET MÉTHODES}

\section{L'essai diallèle}

L'essai diallèle implanté en 1974 sur la station de Barombi-kang est un dispositif $6 \times 6$ quasi complet (sans les autofécondations) en blocs randomisés. II comporte six répétitions, comprenant chacune 12 pieds par famille, réparties sur 2 parcelles distinctes, séparées d'une centaine de mètres: la parcelle A (composée des répétitions 1,2 et 3 ) et la parcelle $B$ (composée des répétitions 4,5 et 6 ). Les espacements entre les arbres sont de 2,5 × $3 \mathrm{~m}$. La densité de plantation (1 330 pieds/ha) est celle qui est recommandée par les services du vulgarisation du Cameroun.

Les géniteurs ont été choisis pour la diversité de leurs origines et pour leur échelonnement dans le classement de sensibilité à la pourriture brune établi par Blaha \& Lotode (1976), en conditions d'infection artificielle. II s'agit des clones SNK10 et SNK413 (Trinitario locaux), ICS95 et ICS84 (Trinitario de Trinidad), IMC67 (Haut amazonien d'lquitos) et UPA134 (descendance de Forastero/Haut amazonien). La sensibilité des géniteurs a été caractérisée par le pourcentage d'infections réussies, selon la méthode de Blaha \& Lotode (1976). Leur classement d'après leur niveau de résistance, du plus faible au plus fort, est le suivant: SNK10 (99), UPA134(94), IMC67 (93), SNK413 (72), ICS95 (37) et ICS84 (24).

Les nombres de pieds survivants pour chacun des croisements en 1987 sont ceux qui peuvent être attendus 13 ans après la mise en place de l'essai (Tableau 1). L'analyse statistique des taux de pertes n'a pas permis de mettre en évidence un effet famille à ce niveau. Les arbres manquants n'ont pas été remplacés.
Tableau I. Effectifs des survivants par familles en 1987.

Géniteurs SNK10 UPA134 IMC67 SNK413 ICS95 ICS84

$\begin{array}{lllllll}\text { SNK10 } & & 50 & 50 & 46 & 60 & 51 \\ \text { UPA134 } & 56 & & 62 & 49 & 62 & 60 \\ \text { IMC67 } & 58 & 63 & & 61 & 51 & 60 \\ \text { SNK413 } & 55 & 48 & 56 & & 50 & 45 \\ \text { ICS95 } & 58 & 63 & 61 & 62 & & 63 \\ \text { ICS84 } & 44 & 48 & 44 & 52 & 48 & \end{array}$

Les effectifs initiaux (1974) étaient de 72 pieds par familles.

\section{Les méthodes de comptage}

Les comptages ont été effectués pied par pied entre les mois de mai et de décembre, pendant trois ans sur la parcelle A (1985, 1986 et 1987) et pendant deux ans sur la parcelle B (1986 et 1987).

Durant ces périodes, les dénombrements de cabosses atteintes de pourriture brune, de cabosses atteintes d'autres affections (wilt physiologique, pourriture noire, dégâts de rongeurs, etc.) et de cabosses mûres récoltées ont été réalisés chaque semaine. A chaque passage, tous les fruits malades ou mûrs étaient enlevés.

\section{Les méthodes d'analyse}

Les analyses, effectuées à partir du cumul des données famille par famille, portent sur la production potentielle (total des fruits mûrs et des fruits pourris), sur la production réelle (total des fruits mûrs effectivement récoltés) et sur les pertes dues à la pourriture brune (proportion des fruits pourris par rapport à la production potentielle)

Les analyses de variance, faites à partir du dispositif en bloc selon la méthode de Griffing (1956), permettent de déterminer 4 paramètres: d'une part l'AGC (aptitude générale à la combinaison) et l'effet materne de chaque géniteur, et, d'autre part, l'ASC (aptitude spécifique à la combinaison) et l'effet réciproque de chacune des combinaisons.

Les estimations des variances de l'ASC et l'AGC ( $\mathrm{S}^{2} \mathrm{Gl}$ et $\mathrm{S}^{2} \mathrm{SI}$ ) permettent d'apprécier les importances respectives de l'ASC et de l'AGC dans l'expression des caractères étudiés selon les clones.

Pour idendifier les différents groupes dans les classements des géniteurs ou des combinaisons, les calculs de la plus petite amplitude significative ont été effectués selon la méthode de Newman \& Keuls:

$$
\mathrm{D}=\frac{q}{\sqrt{2}} \sqrt{\operatorname{Var}(\hat{a} i-\hat{a j})}
$$

D : plus petite amplitude significative âi et âj: effets génétiques considérés (AGC, effet maternel, etc.) 


\section{ANALYSE ET RÉSULTATS}

\section{La production potentielle}

\section{Parcelle A}

La production potentielle totale de juin à décembre sur l'ensemble de la parcelle A pendant 3 ans a été de 57000 cabosses, soit une moyenne de 71,6 cabosses/arbres. A raison de 25 cabosses $/ \mathrm{kg}$ de cacao marchand, le rendement potentiel a donc approché 1,3 tonne $\cdot \mathrm{ha}^{-1} \cdot \mathrm{an}^{-1}$. Les résultats par famille présentent des coefficients de variation importants (Tableau lla).

L'analyse de variance du dispositif montre l'existence d'un effet bloc et d'un effet combinaison (Tableau IIb). L'analyse de variance des aptitudes et des effets indique que l'AGC est la principale source de variation. Cependant les ASC,

Tableau II. Analyse de la production potentielle dans la parcelle A.

a) Tableau des résultats

\begin{tabular}{|c|c|c|c|}
\hline Croisements & Effectifs & Moyenne & Variance \\
\hline SNK10 x UPA134 & 28 & 84,29 & 2449,49 \\
\hline SNK $10 \times$ IMC67 & 24 & 70,96 & 1150,54 \\
\hline SNK10 x SNK413 & 26 & 52,31 & 1681,83 \\
\hline SNK10 $\times 1 C S 95$ & 31 & 65,71 & 2390,27 \\
\hline SNK10 x ICS84 & 22 & 79,36 & 6726,78 \\
\hline UPA134 x SNK10 & 22 & 81,50 & 2448,07 \\
\hline UPA134 x IMC67 & 30 & 81,47 & 2905,32 \\
\hline UPA134 x SNK413 & 20 & 108,45 & 2103,55 \\
\hline UPA134 x ICS95 & 32 & 71,47 & 3211,56 \\
\hline UPA134 x ICS84 & 25 & 68,72 & 2308,60 \\
\hline IMC67 $\times$ SNK10 & 25 & 93,52 & 5287,21 \\
\hline IMC67 x UPA134 & 33 & 65,00 & 2402,97 \\
\hline IMC67 x SNK413 & 22 & 75,36 & 2546,87 \\
\hline IMC67 x ICS95 & 31 & 54,16 & 1051,23 \\
\hline IMC67 x ICS84 & 26 & 69,62 & 2711,85 \\
\hline SNK413 x SNK10 & 25 & 54,16 & 1324,85 \\
\hline SNK413 x UPA134 & 25 & 65,24 & 2200,50 \\
\hline SNK413 x IMC67 & 30 & 65,93 & 2024,26 \\
\hline SNK $413 \times 1$ ICS95 & 27 & 56,96 & 1612,55 \\
\hline SNK413 $\times$ ICS84 & 25 & 61,96 & 2694,36 \\
\hline ICS95 x SNK10 & 29 & 67,00 & 2786,62 \\
\hline ICS95 x UPA134 & 32 & 66,81 & 1609,34 \\
\hline ICS95 x IMC67 & 26 & 50,27 & 1077,97 \\
\hline ICS95 x SNK413 & 22 & 79,82 & 3303,15 \\
\hline ICS95 x ICS84 & 26 & 87,12 & 4754,10 \\
\hline ICS84 x SNK10 & 24 & 96,38 & 3056,98 \\
\hline ICS84 x UPA134 & 30 & 89,73 & 5974,26 \\
\hline ICS84 x IMC67 & 28 & 78,36 & 2892,52 \\
\hline ICS84 x SNK413 & 21 & 71,86 & 9392,60 \\
\hline ICS84 x ICS95 & 29 & 54,62 & 1164,03 \\
\hline Total & 796 & 71,6 & 3018,00 \\
\hline Variance estimée: & 3136,20 & & \\
\hline
\end{tabular}

b) Analyse de variance du dispositif

\begin{tabular}{lccl}
\hline & $\begin{array}{c}\text { Degrés de } \\
\text { liberté }\end{array}$ & $\begin{array}{c}\text { Carrés } \\
\text { moyens }\end{array}$ & $\begin{array}{l}\text { Fde } \\
\text { Fischer }\end{array}$ \\
\hline Total & 89 & & \\
Blocs & 2 & 1796 & $8,34{\left({ }^{* *}\right)}^{* *}$ \\
Familles & 29 & 679 & $3,15\left(^{* *}\right)$ \\
Résidu & 58 & 215 & \\
\hline
\end{tabular}

c) Analyse des aptitudes et des effets

\begin{tabular}{lccc}
\hline & $\begin{array}{c}\text { Degrés de } \\
\text { liberté }\end{array}$ & $\begin{array}{c}\text { Carrés } \\
\text { moyens }\end{array}$ & $\begin{array}{c}\text { Fde } \\
\text { Fischer }\end{array}$ \\
\hline AGC & 5 & 1026 & $4,76\left(^{\star *}\right)$ \\
ASC & 9 & 629 & $2,92\left(^{* *}\right)$ \\
Ef. mat. & 5 & 705 & $3,27\left(^{*}\right)$ \\
Ef. réc. & 10 & 536 & $2,48\left(^{*}\right)$ \\
Résidu & 58 & 215 & \\
\hline
\end{tabular}

d) Classement des géniteurs selon l'AGC

Géniteurs UPA134 ICS84 SNK10 IMC67 SNK413 ICS95

\begin{tabular}{lllllll}
\hline AGC & 7,21 & 5,71 & 3,91 & $-2,76$ & $-5,12$ & $-8,94$ \\
\hline
\end{tabular}

e) Classement des géniteurs selon l'effet maternel

Géniteurs SNK413 SNK10 IMC67 ICS84 UPA134 ICS95

\begin{tabular}{|c|c|c|c|c|c|}
\hline Ef. mat. & 6,90 & 3,82 & $-0,74$ & $-2,24$ & $-3,72$ \\
\hline
\end{tabular}

f) Classement des combinaisons selon l'ASC et l'effet réciproque

\begin{tabular}{|c|c|c|c|}
\hline Croisements & $A S C$ & Croisements & Eff. réciproque \\
\hline $2-4$ & 10,90 & $2-6$ & $10,71-$ \\
\hline $1-6$ & 10,71 & $1-3$ & 6,71 \\
\hline $4-5$ & 8,94 & $1-6$ & 6,06 \\
\hline $1-3$ & 7,14 & $3-4$ & 4,68 \\
\hline $3-4$ & 6,25 & $1-4$ & 3,60 \\
\hline $5-6$ & 2,84 & $3-6$ & 3,56 \\
\hline $1-5$ & 0,43 & $4-5$ & $-0,75$ \\
\hline $1-2$ & 0,15 & $2-5$ & $-1,20$ \\
\hline $3-6$ & $-0,83$ & $2-3$ & $-4,25$ \\
\hline $2-5$ & $-2,81$ & $4-6$ & $-5,18$ \\
\hline $2-3$ & $-3,16$ & $3-5$ & $-5,79$ \\
\hline $2-6$ & $-5,07$ & $1-5$ & $-7,35$ \\
\hline $4-6$ & $-7,65$ & $1-2$ & $-9,03$ \\
\hline $3-5$ & $-9,40$ & $2-4$ & $-14,22$ \\
\hline $1-4$ & $-18,44$ & $5-6$ & $-15,16$ \\
\hline
\end{tabular}

SNK10: 1; UPA134: 2; IMC67: 3; SNK413: 4; ICS95: 5; ICS84: 6

Ef. mat.: effet maternel; Ef. réc.: effet réciproque ": significatif au seuil de $5 \% ; " *$ : significatif au seuil de $1 \%$

les effets maternels et les effets réciproques sont aussi significatifs (Tableau IIc).

Le classement des clones selon leur AGC permet de différencier 3 groupes: les clones UPA134 et ICS84 sont les géniteurs qui présentent les meilleures AGC (Tableau IId). Le classement des combinaisons selon leur ASC permet 
de distinguer deux groupes (Tableau IIf): les croisements UPA $134 \times$ XNK 413 et SNK10xICS84 présentent les meilleures ASC. Les classements des clones selon l'effet maternel ou l'effet réciproque permettent de différencier respectivement deux groupes (Tableau lle,f). L'effet maternel le plus important s'observe pour le géniteur SNK413.

L'estimation des variances de l'AGC et de I'ASC pour UPA134 (S2GI = 44,46, $\left.\mathrm{S}^{2} \mathrm{SI}=13,76\right)$ et $\mathrm{ICS} 84\left(\mathrm{~S}^{2} \mathrm{GI}=25,10 ; \mathrm{S}^{2} \mathrm{SI}=25,05\right)$ montre que l'expression de I'AGC pour UPA134 est moins soumise aux effets d'ASC que pour ICS84.

\section{Parcelle B}

La production potentielle moyenne par arbre de mai à décembre sur l'ensemble de la parcelle $B$ pendant 2 ans a été de 30,3 cabosses/arbre, soit près de 2 fois moindre que dans la parcelle $A$.

Sur cette parcelle, les effets ne sont pas significatifs (Tableau III).

Tableau III. Production potentielle : Analyse de variance dans la parcelle $B$.

\begin{tabular}{lccc}
\hline & $\begin{array}{c}\text { Degrés de } \\
\text { liberté }\end{array}$ & $\begin{array}{c}\text { Carrés } \\
\text { moyens }\end{array}$ & $\begin{array}{c}\text { Fde } \\
\text { Fischer }\end{array}$ \\
\hline Total & 89 & & \\
Blocs & 2 & 290 & 2,28 (NS) \\
Familles & 29 & 134 & 1,05 (NS) \\
Résidu & 58 & 127 & \\
\hline
\end{tabular}

NS: non significatif

\section{La production réelle}

\section{Parcelle A}

La production moyenne de cabosses mûres sur l'ensemble de la parcelle A pendant 3 ans a été de 52,6 cabosses/arbre, soit $900 \mathrm{~kg}$ de cacao marchand $\cdot$ ha $^{-1} \cdot a^{-1}$. Les résultats par famille présentent ici encore des coefficients de variation importants (Tableau IVa).

L'analyse de variance du dispositif montre l'existence d'un effet bloc et d'un effet famille (Tableau IVb). L'analyse des aptitudes et des effets indique que l'AGC est la principale source de variation. Les ASC, les effets maternels et les effets réciproques sont également significatifs (Tableau IVc).

Le classement des clones selon leur AGC permet de différencier trois groupes (Tableau IVd).
Tableau IV. Analyse de la production réelle dans la parcelle A.

a) Tableau des résultats

\begin{tabular}{|c|c|c|c|}
\hline Croisements & Effectifs & Moyenne & Variance \\
\hline SNK10 x UPA134 & 28 & 66,14 & 1729,55 \\
\hline SNK10 x IMC67 & 24 & 41,21 & 312,33 \\
\hline SNK10 $\times$ SNK413 & 26 & 40,46 & 918,94 \\
\hline SNK10 x ICS95 & 31 & 38,58 & 898,44 \\
\hline SNK10 x ICS84 & 22 & 59,64 & 3540,14 \\
\hline UPA134 x SNK10 & 22 & 62,32 & 965,40 \\
\hline UPA134 x IMC67 & 30 & 60,57 & 1562,11 \\
\hline UPA134 x SNK413 & 20 & 95,75 & 1889,99 \\
\hline UPA134 x ICS95 & 32 & 49,84 & 1499,32 \\
\hline UPA134 x ICS84 & 25 & 53,68 & 1481,34 \\
\hline IMC67 x SNK10 & 25 & 59,04 & 2531,72 \\
\hline IMC67 x UPA134 & 33 & 51,52 & 1415,52 \\
\hline IMC67 x SNK413 & 22 & 57,32 & 1672,67 \\
\hline IMC67 x ICS95 & 31 & 38,45 & 505,15 \\
\hline IMC67 x ICS84 & 26 & 46,35 & 816,76 \\
\hline SNK413 x SNK10 & 25 & 36,96 & 661,72 \\
\hline SNK413 x UPA134 & 25 & 55,08 & 1582,95 \\
\hline SNK413 x IMC67 & 30 & 53,17 & 1183,07 \\
\hline SNK413 x ICS95 & 27 & 39,41 & 820,09 \\
\hline SNK413 $\times$ ICS84 & 25 & 48,64 & 1308,15 \\
\hline ICS95 x SNK10 & 29 & 37,52 & 564,94 \\
\hline ICS95 x UPA134 & 32 & 53,84 & 1001,82 \\
\hline ICS95 x IMC67 & 26 & 36,85 & 584,89 \\
\hline ICS95 x SNK413 & 22 & 60,09 & 1693,26 \\
\hline ICS95 x ICS84 & 26 & 59,85 & 2014,82 \\
\hline ICS84 x SNK10 & 24 & 68,00 & 1393,50 \\
\hline ICS84 x UPA134 & 30 & 78,27 & 4789,00 \\
\hline ICS84 x IMC67 & 28 & 63,29 & 2128,28 \\
\hline ICS84 x SNK413 & 21 & 43,71 & 2595,63 \\
\hline ICS84 x ICS95 & 29 & 37,69 & 516,97 \\
\hline $\begin{array}{l}\text { Total } \\
\text { Variance estimée: }\end{array}$ & $\begin{array}{r}796 \\
1696,44\end{array}$ & 52,59 & 1632,50 \\
\hline
\end{tabular}

b) Analyse de variance du dispositif

\begin{tabular}{lccr}
\hline & $\begin{array}{c}\text { Degrés de } \\
\text { liberté }\end{array}$ & $\begin{array}{c}\text { Carrés } \\
\text { moyens }\end{array}$ & $\begin{array}{l}\text { Fde } \\
\text { Fischer }\end{array}$ \\
\hline Total & 89 & & \\
Blocs & 2 & 1796 & $12,64\left(^{\star \star}\right)$ \\
Familles & 29 & 603 & $5,15\left(^{\star \star}\right)$ \\
Résidu & 58 & 117 & \\
\hline
\end{tabular}

c) Analyse des aptitudes et des effets

\begin{tabular}{lcrc}
\hline & $\begin{array}{c}\text { Degrés de } \\
\text { liberté }\end{array}$ & $\begin{array}{c}\text { Carrés } \\
\text { moyens }\end{array}$ & $\begin{array}{c}\text { Fde } \\
\text { Fischer }\end{array}$ \\
\hline AGC & 5 & 1381 & $11,79\left(^{* *}\right)$ \\
ASC & 9 & 442 & $3,77\left(^{\star *}\right)$ \\
Ef. mat. & 5 & 387 & $3,30\left(^{*}\right)$ \\
Ef. réc. & 10 & 466 & $3,98\left(^{\star}\right)$ \\
Résidu & 58 & 117 & \\
\hline
\end{tabular}


d) Classement des géniteurs selon l'AGC

\begin{tabular}{|c|c|c|c|c|c|}
\hline Géniteurs & UPA134 & ICS 84 & $S N K 413$ & SNK1a & MC67 ICS95 \\
\hline AGC & 12,13 & 4,36 & $-0,78$ & $-2,10$ & $-10,27$ \\
\hline
\end{tabular}

e) Classement des géniteurs selon l'effet maternel

Géniteurs SNK413 SNK10 IMC67 ICS84 UPA134 ICS95

\begin{tabular}{|c|c|c|c|c|c|c|}
\hline Ef. mat. & 5,49 & 1,70 & $-0,31$ & $-1,92$ & $-1,98$ & $-3,60$ \\
\hline
\end{tabular}

f) Classement des combinaisons selon l'ASC et l'effet réciproque

\begin{tabular}{|c|c|c|c|}
\hline Croisements & $A S C$ & Croisements & Eff. réciproque \\
\hline $1-6$ & $11,36-7$ & $2-6$ & 10,427 \\
\hline $2-4$ & 10,61 & $1-3$ & 7,50 \\
\hline $4-5$ & 6,40 & $3-6$ & 6,60 \\
\hline $3-4$ & 6,26 & $3-4$ & 4,07 \\
\hline $5-6$ & 2,07 & $1-6$ & 2,76 \\
\hline $1-2$ & 1,39 & $1-4$ & 1,52 \\
\hline $1-3$ & 1,09 & $2-5$ & 1,09 \\
\hline $3-6$ & 0,58 & $4-5$ & $-0,15$ \\
\hline $1-5$ & $-1,99$ & $2-3$ & $-1,69$ \\
\hline $3-5$ & $-2,50$ & $3-5$ & $-4,86$ \\
\hline $2-6$ & $-2,59$ & $1-5$ & $-5,88$ \\
\hline $2-5$ & $-3,97$ & $1-2$ & $-5,91$ \\
\hline $2-3$ & $-5,43$ & $5-6$ & $-9,80$ \\
\hline $4-6$ & $-11,42$ & $4-6$ & $-9,99$ \\
\hline $1-4$ & $-11,85$ & $2-4$ & $-15,74$ \\
\hline
\end{tabular}

SNK10: 1; UPA134: 2; IMC67: 3; SNK413: 4; ICS95: 5; ICS84: 6 Ef. mat:: effet maternel; Eł. réc.: effet réciproque *: significatif au seuil de $5 \%$; ** : significatif au seuil de $1 \%$

Le clone UPA134, qui présente la meilleure AGC pour ce critère, constitue un groupe à lui seul. Les classements selon l'ASC, l'effet maternel et l'effet réciproque permettent respectivement de mettre en évidence l'existence de deux groupes distincts (Tableau IVe, f). II faut noter l'effet maternel particulièrement marqué pour le géniteur SNK413.

L'estimation des variances de l'AGC et de I'ASC pour UPA134 (S'GI = 143,06; $\mathrm{S}^{2} \mathrm{SI}=$ $27,01)$ et $S N K 413\left(S^{2} G I=-3,45 ; S^{2} S I=101,30\right)$ montre que l'expression de I'AGC pour UPA134 est moins soumise aux effets d'ASC que pour SNK413.

\section{Parcelle B}

La production de cabosses mûres sur l'ensemble de la parcelle $B$ pour les 2 années de récolte (17,9 cabosses/arbre) est nettement moins importante que celle de la parcelle $A$.

L'analyse de variance du dispositif indique l'existence d'un effet bloc et d'un effet combinai- son (Tableau Va). L'analyse des aptitudes et des effets montre que la principale source de variation est l'effet réciproque (Tableau $\mathrm{Vb}$ ). La comparaison des croisements selon le test de Newman et Keuls ne permet pas de distinguer plusieurs groupes parmi les combinaisons.

\section{Les pertes provoquées par la pourriture brune}

\section{Parcelle A}

Le pourcentage moyen de cabosses pourries pour les 3 années a été de $26,4 \%$. Ce taux d'attaque est faible pour la région, où les pertes avoisinent souvent les $70 \%$. La variabilité intrafamille est ici encore importante (Tableau Vla). Les individus d'une même famille présentent souvent des pourcentages de pertes très différents.

L'analyse de variance du dispositif indique l'existence d'un effet bloc et d'un effet famille (Tableau VIb). L'analyse des aptitudes et des effets montre que l'AGC est la principale source de variation (Tableau VIC). Les ASC et les effets réciproques sont également significatifs.

Le classement des clones selon l'AGC permet de différencier 3 groupes parmi les géniteurs. Le clone UPA134, qui constitue un groupe à lui seul, possède la meilleure $A G C$ pour la résistance au champ (Tableau VId).

Tableau V. Analyse de la production réelle dans la parcelle B.

a) Analyse de variance du dispositif

\begin{tabular}{lccc}
\hline & $\begin{array}{c}\text { Degrés de } \\
\text { liberté }\end{array}$ & $\begin{array}{c}\text { Carrés } \\
\text { moyens }\end{array}$ & $\begin{array}{c}\text { Fde } \\
\text { Fischer }\end{array}$ \\
\hline Total & 89 & & \\
Blocs & 2 & 159 & $4,90\left(^{\star \star}\right)$ \\
Familles & 29 & 63 & $1,95\left(^{*}\right)$ \\
Résidu & 58 & 32 & \\
\hline
\end{tabular}

b) Analyse des aptitudes et des effets

\begin{tabular}{lccc}
\hline & $\begin{array}{c}\text { Degrés de } \\
\text { liberté }\end{array}$ & $\begin{array}{c}\text { Carrés } \\
\text { moyens }\end{array}$ & $\begin{array}{c}\text { Fde } \\
\text { Fischer }\end{array}$ \\
\hline AGC & 5 & 73 & 2,25 (NS) \\
ASC & 9 & 63 & 1,95 (NS) \\
Ef. mat. & 5 & 31 & $0,95(\mathrm{NS})$ \\
Ef. réc. & 10 & 74 & $2,28\left(^{\star}\right)$ \\
Résidu & 58 & 32 & \\
\hline
\end{tabular}

Ef. mat: effet maternel; Ef. réc.: effet réciproque (NS): non significatif; $\left({ }^{*}\right)$ : significatif au seuil de $5 \%$;

(“*): significatif au seuil de $1 \%$ 
Tableau VI. Analyse des pertes dans la parcelle A.

a) Tableau des résultats

\begin{tabular}{|c|c|c|c|}
\hline Croisements & Effectifs & Moyenne & Variance \\
\hline SNK10 x UPA134 & 28 & 0,21 & 0,04 \\
\hline SNK10 x IMC67 & 24 & 0,35 & 0,04 \\
\hline SNK10 x SNK413 & 26 & 0,18 & 0,04 \\
\hline SNK10 1 ICS95 & 31 & 0,35 & 0,06 \\
\hline SNK10 x ICS84 & 22 & 0,20 & 0,03 \\
\hline UPA134 x SNK10 & 22 & 0,18 & 0,03 \\
\hline UPA134 x IMC67 & 30 & 0,23 & 0,03 \\
\hline UPA134 x SNK413 & 20 & 0,12 & 0,02 \\
\hline UPA134 x ICS95 & 32 & 0,24 & 0,05 \\
\hline UPA134 $\times$ ICS 84 & 25 & 0,20 & 0,03 \\
\hline IMC67 × SNK10 & 25 & 0,33 & 0,05 \\
\hline IMC67 x UPA134 & 33 & 0,14 & 0,02 \\
\hline IMC67 $\times$ SNK413 & 22 & 0,21 & 0,03 \\
\hline IMC67 x ICS95 & 31 & 0,25 & 0,04 \\
\hline IMC67 x ICS84 & 26 & 0,26 & 0,06 \\
\hline SNK413 x SNK10 & 25 & 0,30 & 0,08 \\
\hline SNK413 x UPA134 & 25 & 0,14 & 0,01 \\
\hline SNK413 x IMC67 & 30 & 0,15 & 0,02 \\
\hline SNK $413 \times 1$ ICS95 & 27 & 0,27 & 0,04 \\
\hline SNK $413 \times 1 \mathrm{CS} 84$ & 25 & 0,18 & 0,04 \\
\hline ICS95 x SNK10 & 29 & 0,29 & 0,06 \\
\hline ICS95 x UPA134 & 32 & 0,16 & 0,02 \\
\hline ICS95 x IMC67 & 26 & 0,23 & 0,04 \\
\hline ICS95 x SNK413 & 22 & 0,22 & 0,03 \\
\hline ICS95 x ICS84 & 26 & 0,24 & 0,04 \\
\hline ICS 84 x SNK10 & 24 & 0,28 & 0,06 \\
\hline ICS $84 \times$ UPA134 & 30 & 0,11 & 0,01 \\
\hline ICS84 x IMC67 & 28 & 0,19 & 0,03 \\
\hline ICS84 x SNK413 & 21 & 0,27 & 0,04 \\
\hline ICS84 x ICS95 & 29 & 0,32 & 0,02 \\
\hline Total & 796 & 0,225 & 0,041 \\
\hline Variance estimée: & 0,043 & & \\
\hline
\end{tabular}

b) Analyse de variance du dispositif

\begin{tabular}{lccc}
\hline & $\begin{array}{c}\text { Degrés de } \\
\text { liberté }\end{array}$ & $\begin{array}{c}\text { Carrés } \\
\text { moyens }\end{array}$ & $\begin{array}{l}\text { Fde } \\
\text { Fischer }\end{array}$ \\
\hline Total & 89 & & \\
Blocs & 2 & 769 & $14,36\left(^{* *}\right.$ \\
Familles & 29 & 204 & $3,79{\left({ }^{*}\right)}^{*}$ \\
Résidu & 58 & 54 & \\
\hline
\end{tabular}

c) Analyse des aptitudes et des effets

\begin{tabular}{lcrc}
\hline & $\begin{array}{c}\text { Degrés de } \\
\text { liberté }\end{array}$ & $\begin{array}{c}\text { Carrés } \\
\text { moyens }\end{array}$ & $\begin{array}{c}F d e \\
\text { Fischer }\end{array}$ \\
\hline AGC & 5 & 702 & $13,02\left(^{* *}\right)$ \\
ASC & 9 & 121 & $2,25\left(^{* *}\right)$ \\
Ef. mat. & 5 & 40 & $0,74(\mathrm{NS})$ \\
Ef. réc. & 10 & 112 & $2,07\left(^{*}\right)$ \\
Résidu & 58 & 54 & \\
\hline
\end{tabular}

d) Classement des géniteurs selon l'AGC

\begin{tabular}{|c|c|c|c|c|c|c|}
\hline Géniteurs & UPA13 & SNK413 & $1 C S 8$ & IMC67 & ICS95 & NK10 \\
\hline$A G C$ & $-8,52$ & $-3,85$ & 0,09 & 1,98 & 4,16 & 6,13 \\
\hline
\end{tabular}

e) Classement des géniteurs selon l'ASC et l'effet réciproque

\begin{tabular}{cccc}
\hline Croisements & ASC & Croisements & Eff. réciproque \\
\hline $1-6$ & $-5,72$ & $3-6$ & $-7,14$ \\
$3-5$ & $-5,14$ & $1-3$ & $-5,14$ \\
$1-4$ & $-2,56$ & $2-3$ & $-2,94$ \\
$3-4$ & $-2,31$ & $1-2$ & $-2,15$ \\
$2-4$ & $-1,87$ & $2-6$ & $-1,00$ \\
$1-2$ & $-1,09$ & $3-5$ & $-0,90$ \\
$3-6$ & $-0,97$ & $2-5$ & $-0,84$ \\
$2-6$ & $-0,71$ & $5-6$ & $-0,80$ \\
$4-5$ & $-0,07$ & $4-5$ & $-0,56$ \\
$5-6$ & 0,58 & $3-4$ & $-0,03$ \\
$2-5$ & 1,77 & $1-6$ & 1,20 \\
$2-3$ & 1,90 & $1-5$ & 1,50 \\
$1-5$ & 2,85 & $2-4$ & 2,62 \\
$1-3$ & 6,52 & $1-4$ & 4,58 \\
$4-6$ & 6,52 & $4-6$ & 7,74 \\
\hline
\end{tabular}

SNK10: 1; UPA 134: 2; IMC67: 3: SNK413: 4; ICS95: 5; ICS84: 6

Ef. mat.: effet maternel; Ef. réc.: effet réciproque; (NS): non significatif; ": significatif au seuil de $5 \%$; ${ }^{* *}$ : significatif au seuil de $1 \%$

La comparaison des croisements d'après I'ASC ou l'effet réciproque selon le test de Newman \& Keuls ne permet pas de distinguer plusieurs groupes parmi les combinaisons (Tableau Vle).

L'estimation des variances de l'AGC et de l'ASC pour le clone UPA134 (S2GI = 70,73; S2SI $=3,67)$ montre que ce géniteur transmet son $A G C$ de manière relativement uniforme. Pour le clone SNK413 ( $\mathrm{S}^{2} \mathrm{GI}=12,9 ; \mathrm{S}^{2} \mathrm{SI}=8,7$ ), l'écart est plus faible. L'expression de sa valeur AGC est plus hétérogène, en raison d'effets spécifiques plus importants.

\section{Parcelle B}

L'analyse de variance du dispositif indique l'existence d'un effet bloc et d'un effet famille (Tableau VIla). L'analyse des aptitudes et des effets montre que I'AGC est la principale source de variation (Tableau Vllb).

Le classement des clones d'après l'AGC permet de différencier 3 groupes parmi les géniteurs. Comme dans la parcelle $A$, c'est le clone UPA134 qui possède la meilleure AGC pour la transmission du caractère de résistance. 
Tableau VII. Analyse des pertes dans la parcelle B.

a) Analyse de variance du dispositif

\begin{tabular}{lccl}
\hline & $\begin{array}{c}\text { Degrés de } \\
\text { liberté }\end{array}$ & $\begin{array}{c}\text { Carrés } \\
\text { moyens }\end{array}$ & $\begin{array}{l}\text { Fde } \\
\text { Fischer }\end{array}$ \\
\hline Total & 89 & & \\
Blocs & 2 & 660 & $6,03{\left(\left(^{* *}\right)\right.}^{\text {Familles }}$ \\
Résidu & 9 & 232 & $2,12\left(^{*}\right)$ \\
\hline
\end{tabular}

b) Analyse des aptitudes et des effets

\begin{tabular}{lccc}
\hline & $\begin{array}{c}\text { Degrés de } \\
\text { liberté }\end{array}$ & $\begin{array}{c}\text { Carrés } \\
\text { moyens }\end{array}$ & $\begin{array}{c}\text { Fde } \\
\text { Fischer }\end{array}$ \\
\hline AGC & 5 & 848 & $7,74\left(^{* *}\right)$ \\
ASC & 9 & 38 & $0,35(\mathrm{NS})$ \\
Ef. mat. & 5 & 239 & $2,18(\mathrm{NS})$ \\
Ef. réc. & 10 & 97 & $0,88(\mathrm{NS})$ \\
Résidu & 58 & 110 & \\
\hline
\end{tabular}

c) Classement des géniteurs selon l'AGC

Géniteurs UPA134 SNK413 ICS84 IMC67 ICS95 SNK10

\begin{tabular}{lllllll}
\hline AGC & $-8,73$ & $-2,72$ & $-2,43$ & 0,85 & 5,68 & 7,36 \\
\hline & & & &
\end{tabular}

Ef. mat.: effet maternel; Ef. réc.: effet réciproque; (NS): non significatif; ": significatif au seuil de 5\%;"* : significatif au seuil de $1 \%$

\section{DISCUSSION ET CONCLUSIONS}

L'observation des infections naturelles sur les cabosses dans l'essai diallèle de Barombi-kang a permis de mettre en évidence des différences significatives entre les croisements, malgré une variabilité intrafamille relativement importante. La structure génétique des origines travaillées étant de type hétérozygote, il est normal d'observer une forte variabilité intrafamille pour les caractères mesurés, et ce d'autant plus que ceux-ci ne possèdent pas un déterminisme génétique simple.

L'étude de la production potentielle pendant la période de grande récolte (plus de $90 \%$ de la récolte totale) permet d'évaluer la productivité des familles sans tenir compte de la pression de la maladie. Cette dernière apparait essentiellement liée à des effets d'AGC. Dans leur ensemble, les croisements avec le clone UPA134 permettent le développement d'un plus grand nombre de fruits. En proportion, les effets spécifiques sont relativement faibles. Les familles issues du clone SNK413 sont en général moins productives. Cependant, il faut noter que ce clone se distingue des autres géniteurs par les ASC de certains croisements dont il est l'un des parents et un effet maternel particulièrement marqués.

Le rendement final en cacao marchand ne dépend pas uniquement du nombre de fruits arrivés à maturité, mais aussi du nombre et de la taille des fèves dans chaque fruit. Ces caractéristiques sont en général de bon niveau pour le clone UPA134, qui a déjà été choisi au Cameroun comme père ou mère dans plusieurs champs semenciers. Néanmoins, une confirmation définitive sera apportée par des travaux complémentaires sur les caractéristiques des fruits dans chaque famille, actuellement en cours de réalisation.

Par ailleurs, les descendances du clone UPA134 ont subi des pertes inférieures aux autres familles. Le même phénomène est observé pour les descendances de SNK413, mais à un moindre niveau.

Ces différences dans les taux de fruits pourris sont essentiellement liées aux AGC des géniteurs. Les caractères génétiques en cause dans l'expression de cette résistance sont donc transmis surtout de manière additive. La prédiction de la valeur des descendances de UPA134 est plus facile que dans le cas de SNK413, en raison d'effets d'ASC plus faibles.

Le clone UPA134 s'était révélé très sensible aux infections artificielles sur cabosses. Or, les résultats obtenus ici indiquent que ce géniteur possède une forte $A G C$ pour un ou plusieurs caractère(s) qui entraîne(nt) une réduction des pertes durant l'épidémie.

Plus généralement, les positions respectives des six clones parents, selon leurs niveaux de résistance évalués à la suite d'infections artificielles, n'apparaissent pas directement corrélées à leur AGC pour une moindre sensibilité au champ, déterminée à partir du comportement de leurs descendances. Le test non paramétrique de Friedman montre que les deux classements sont indépendants $\left(\chi^{2}=5,71\right.$, d.d.. . $\left.=5\right)$.

Une nouvelle parcelle expérimentale avec les clones parents en randomisation totale a été mise en place dans la même station en 1982. Mais la productivité de cette plantation est encore insuffisante pour étudier leur comportement dans les conditions naturelles d'infection.

En théorie, les valeurs des AGC pour la résistance ne sont pas nécessairement liées au niveau de résistance des parents. Toutefois, la variance importante due à l'AGC prédit plutôt une forte corrélation entre le niveau de résistance des parents et celui de leurs descendances.

L'hypothèse la plus probable pour expliquer cette apparente contradiction est qu'il existe un ou plusieurs caractère(s) d'esquive ou de résis- 
tance jouant un rôle important dans la réussite naturelle de l'infection, qui sont involontairement masqués (ou surmontés) lors des infections artificielles, telles qu'elles ont été pratiquées par Blaha \& Lotode (1976).

Une esquive de la maladie peut, en effet, être due à des modalités de production particulières: précocité de mise à fruit, dispersion des fruits, conformation originale des pieds, etc. L'existence d'un tel phénomène ne peut pas être complètement écartée, bien que l'observation visuelle n'ait pas permis d'établir de différences notables entre les descendances. Une autre explication possible est que la quantité de fruits produits par les arbres aurait une incidence déterminante sur le déroulement de l'épidémie. Toutefois, le test non paramètrique de Friedman montre que les classements en fonction des AGC pour la production et les taux de pourriture brune sont indépendants $\left(\chi^{2}=8,86\right.$, d.d.I. $\left.=5\right)$.

La nature du (des) caractère(s) spécifique(s) de l'hôte qui se tradui(sen)t au champ par une moindre incidence de la maladie reste donc encore à identifier. Cependant, la modification apparente la plus importante, inhérente au protocole d'infection artificielle par rapport au processus d'infection naturelle, est la mise en place d'un godet de pâte à modeler pour assurer le maintien de la goutelette infectieuse au contact de la cabosse. II est possible qu'une des barrières naturelles des fruits à la maladie se situe à ce niveau (rapidité de l'évaporation du film d'eau à la surface des fruits, cuticule plus ou moins hydrophobe, difficulté de fixation des zoospores, etc.). Des recherches doivent être entreprises pour étudier l'influence potentielle de ce type de facteurs dans la résistance ou la sensibilité des clones.

D'autres part, les différences de comportement entre les familles paraissent plus prononcées dans la parcelle $A$ que dans la parcelle $B$. Ce phénomène est probablement la conséquence d'une moindre production dans la parcelle $B$. Lorsque l'analyse est réalisée sur l'ensemble des deux parcelles $A$ et $B$, les résultats obtenus sont similaires à ceux qui ont été observés dans la seule parcelle $A$.

Les différences de production entre les deux parcelles ont été constatées dès l'entrée en production des arbres. II semble qu'elles soient dues à un effet de terrain, qui n'a pourtant pas été relevé au moment de la plantation. Les cacaoyers sont, en général, plus vigoureux dans la parcelle $B$, mais ils portent moins de fruits que dans la parcelle A.

Enfin, le taux de fruits malades a été particulièrement bas sur l'ensemble de la plantation
$(26,4 \%)$. Dans la même station expérimentale, les autres cacaoyères ont subi des pertes moyennes de l'ordre de $60 \%$, même celles qui étaient de densité équivalente et soumises aussi à une récolte sanitaire hebdomadaire. II est possible que la différence observée soit consécutive à la présence de pieds partieliement résistants dans le diallèle. En effet, un faible niveau de résistance intrinsèque peut avoir un effet important sur le déroulement de la maladie, par exemple en freinant la multiplication de l'inoculum. Si tel était le cas, l'effet dépressif sur l'épidémie devrait être encore accentué dans une plantation uniquement composée de cacaoyers partiellement résistants. Par ailleurs, le déterminisme apparemment polygénique de cette résistance permet d'espérer qu'elle ne sera pas facilement contournée par le parasite. La confirmation de ces deux hypothèses ouvrirait des perspectives très encourageantes quant à l'efficacité potentielle de la lutte génétique contre la pourriture brune des cabosses.

\section{REMERCIEMENTS}

Les auteurs remercient Monsieur Ayouk Takem, Directeur de I'IRA, Monsieur Awemo, Chef de la Station de Barombi-kang et Monsieur Kitio, Chef de l'équipe cacao de la Station de Barombi-kang, qui ont permis la réalisation de ces travaux.

\section{RÉFÉRENCES}

Amponsah J.D. \& Asare Nyako A. (1973) Glasshouse method for screenning cocoa (Theobroma cacao L.) for resistance to Black Pod caused by Phytophthora palmivora (Butl.) Butl. Trop. Agric. Trinidad 50, 143152

Bakala J. (1979) Répartition des types morphologiques de Phytophthora palmivora (Butl.) Butl. au Cameroun. 7e Conf. Int. Rec. Cac., Douala, Cameroun, Alliance des pays producteurs de cacao, Londres (U.K.), 297-301

Blaha G. \& Lotode R. (1976) Un caractère primordial de sélection du cacaoyer au Cameroun: la résistance à la pourriture brune des cabosses, Café Cacao Thé, $X X, 97-116$

Despréaux D., Cambrony D., Clément D., Nyasse S. \& Partiot M. (1987) Etude de la pourriture brune des cabosses du cacaoyer au Cameroun: définition de nouvelles méthodes de lutte, 10 e Conf. Int. Rec. Cac., Saint Domingue, Alliance des pays producteurs de cacao, Londres (U.K.), 407-412

Gregory P.H. \& Maddisson A.C. (1984) Epidemiology of Phytophthora on cocoa in Nigeria, Cocoa Grower Bull., Londres (U.K.) 34, 2-14

Griffin M.J. (1977) Cocoa phytophthora workshop, 2426 mai 1976, Rothamsted experimental station (U.K.), PANS 23, 107-110 
Griffing B. (1956) Concept of general and specific combining ability in relation to diallel systems, Aust. $J$. Biol. Sci. 9, 463-473

Lawrence J.S. (1978) Evaluation of methods of assessing resistance of Theobroma cacao $L$. cultivars and hybrids to Phytophthora palmivora (Butl.) Butl., Bol. técnico 62, Comissao executiva do plano da lavoura cacaueira, Bahia, Brésil

Nwaga D. (1984) Contribution à l'amélioration génétique de la résistance du cacaoyer (Theobroma cacao L.) à la pourriture brune des cabosses dues à Phytophthora spp.: mise au point d'un test précoce de la résistance, DEA Ecophysiologie et Génétique végatales, Univ. de Rennes I

Partiot M. (1975) La résistance horizontale du cacaoyer au Phytophthora sp.: contribution à l'étude de son évaluation, de son amélioration et de son utilisation. Thèse 3 e cycle, Univ. Paris-Sud, Orsay

Tarjot M. (1977) A study of Black Pod in the cocoa tree due to Phytophthora palmivora. A first adaptation to Cameroon conditions of an early sensitivity test on young seedlings. 6e Conf. Int. Rech. Cac., Caracas, Vénézuéla, Alliance des pays producteurs de cacao, Londres (U.K.), 214-217 